\title{
Optical conductance of waveguides built into finite photonic crystals
}

\author{
S. Albaladejo a) and J. J. Sáenz \\ Departamento de Física de la Materia Condensada and Instituto "Nicolás Cabrera," Universidad \\ Autónoma de Madrid, E-28049 Madrid, Spain \\ M. Lester \\ IFAS, Facultad de Ciencias Exactas, UNCPBA, Pinto 399, 7000 Tandil, Argentina and CONICET, \\ Rivadavia 1917, 1033 Buenos Aires, Argentina \\ L. S. Froufe-Pérez \\ Laboratoire EM2C, CNRS, Ecole Centrale Paris, 92295 Châtenay-Malabry, France \\ A. García-Martín \\ Instituto de Microelectrónica de Madrid, CSIC, Isaac Newton 8, Tres Cantos, 28760 Madrid, Spain
}

(Received 22 March 2007; accepted 28 June 2007; published online 6 August 2007)

\begin{abstract}
The concept of optical conductance is introduced in order to characterize the transport properties of waveguides built into finite photonic crystals. The conductance is given by the integral of the transmission cross section as a function of the incoming angle. This concept is illustrated by exact calculations of the light-guiding properties of a waveguide built into a photonic crystal slab based on a square lattice of dielectric cylinders in air. In analogy with their electronic counterparts, the optical conductance of a waveguide is quantized and thus provides a direct measure of the number of guided modes supported by the system. (C) 2007 American Institute of Physics.
\end{abstract}

[DOI: 10.1063/1.2761223]

Photonic crystals (PCs) provide a set of unique options to control the electromagnetic states and light transport without radiation and absorption losses. ${ }^{1-3}$ Their ability to guide light on the wavelength scale ${ }^{4,5}$ makes them especially suitable to miniaturization of optical components. Line defects in photonic crystals are receiving considerable attention because their waveguiding mechanism is fundamentally different from conventional dielectric waveguides. The existence of a band gap prohibits radiation of energy through the crystal and, as a consequece, the confinement of electromagnetic waves in photonic crystal waveguides (PCWs) resembles the electron wave confinement in nanowires.

In the study of electron transport in nano- and mesoscopic systems, the discovery of the quantization of conductance of narrow constrictions in fabricated semiconductor structures $^{6}$ has had a profound impact. For a long, defectfree, constriction the conductance is proportional to the integer number of propagating modes or channels and increases in steps of $G_{0}=2 e^{2} / h$ each time a channel opens up as its width increases. This result, independent of any material detail, plays a key role in the understanding of electron transport on the wavelength scale: from semiconductor structures ${ }^{6}$ down to nanowires and atomic-scale metallic contacts. ${ }^{7}$ Surprisingly, in contrast with the study of their electronic counterparts, the concept of optical conductance of a PCW has not received special attention.

In this letter, we introduce the concept of optical conductance in order to characterize the transport properties of photonic crystal waveguides. Conductance quantization (CQ)has its optical analogue in the stepwise increase of the transmission cross section of a slit illuminated by diffusive monochromatic light. ${ }^{8}$ The diffuse illumination is equivalent to the isotropic momentum distribution of the incoherent electron

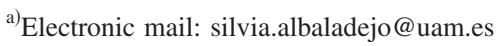

waves incident on a constriction. This is in contrast with the standard approach to analyze the scattering and diffraction characteristics of PCWs. These systems are usually studied assuming spatial coherent illumination (from plane wave or dipole sources). ${ }^{4,5,9,10}$ In general, the ratio of transmitted power to incident flux (the transmission cross section) depends in a complicated way on the angle of incidence, PCW shape, and size as well as on the details of the mode conversion at the aperture. Diffuse illumination simplifies this complex dependence. ${ }^{8}$ Here we show that the conductance can be measured with coherent illumination by integrating the transmission cross section as a function of the incoming angle. In contrast with other "transmission" coefficients, the conductance is an intrinsic property of the PCW and can be calculated and, eventually, measured unambiguously.

To illustrate the concept of optical conductance of a PCW, we present exact calculations of the photonic and waveguiding properties of two-dimensional PC slab waveguides. The optical conductance of the PCW is analyzed as a function of the width of the waveguide, wavelength, and crystal size. As we will show, in analogy with their electronic counterparts, the optical conductance of a waveguide is quantized and provides a direct measure of the number of guided modes supported by the system. Most of the work on PCWs is based on the waveguiding properties of single or multiple line defects in an otherwise perfect crystal. In a different approach, the walls of two PCs provide an equivalent confinement of light, ${ }^{9}$ similar to that provided by perfect metals. With the aim of studying and characterizing the propagation of electromagnetic modes in a PCW, we consider a dielectric PCW made up of two identical finite photonic crystals. In this way we obtain a PCW with a variable width $W$ (see Fig. 1) just by changing the gap between the two PCs, in analogy with the slit problem. ${ }^{8}$ Each PC is an array of infinitely long cylinders parallel to the $z$ axis, embedded in air, and forming a square lattice with lattice con- 


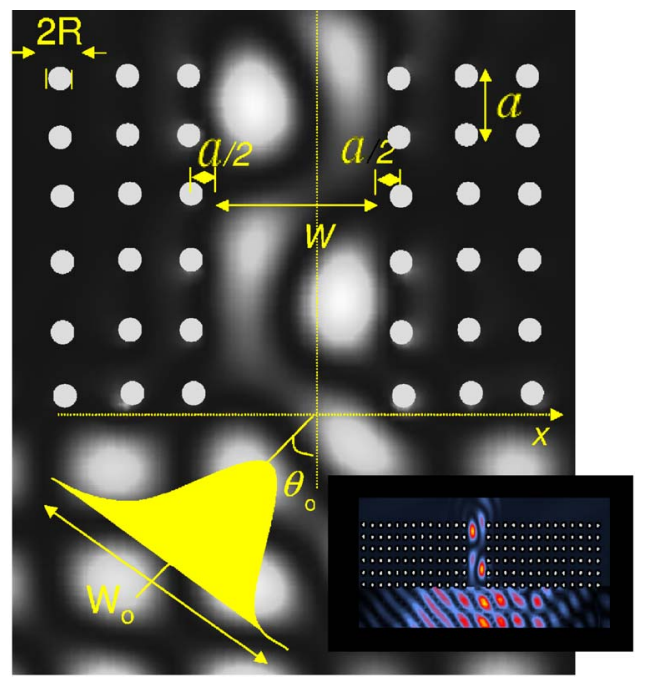

FIG. 1. (Color online) Zoom out of the waveguide region including the definition of relevant parameters. Calculations correspond to Si nanorods in air with $a=0.6 \mu \mathrm{m}, R / a=0.16, \lambda=1.33 \mu \mathrm{m}$, and $\theta_{0}=45^{\circ}$. In this particular case $W=2 a$. Inset: scheme of the structure of a photonic crystal waveguide of variable width illuminated by a Gaussian beam. The intensity map corresponds to actual calculations of the electric field intensity (with the electric field perpendicular to the plane of the figure).

stant $a$. Each finite crystal has $M$ rows and $N$ columns along the $x$ and $y$ axes, respectively. The band structure and scattering properties of a square lattice of dielectric rods or nanopillars have been discussed before. ${ }^{3,11-16}$ For an infinite square lattice of cylinders in air, band gaps only open up for TM or $s$ polarization (with the electric field along the cylinder axis), while for the TE polarization no band gaps are expected. ${ }^{3}$ We shall then focus on the scattering of TM polarized electromagnetic waves. We will also consider silicon dielectric rods as a typical dielectric material. Our results, however, are general and independent of the nature of the PCW.

A Gaussian beam, ${ }^{17} E_{\text {inc }}(\mathbf{r})$ of half-width $W_{0}$, focused on the entrance of the waveguide $(x=y=0)$, is incident upon the system from $y<0$; its vector wave $\mathbf{k}_{0}=k \sin \theta_{0} \mathbf{u}_{x}+k \cos \theta_{0}$ $\mathbf{u}_{y}$ (with $k=\omega / c=2 \pi / \lambda$ ) is contained in the $x-y$ plane.

In order to compute numerically the scattered field, we use the coupled dipole method ${ }^{18,19}$ which allows us to solve the electromagnetic scattering problem rigorously. We consider subwavelength silicon cylindrical rods with a ratio $R / a=0.16$ between radius $R$ and lattice spacing $a=0.6 \mu \mathrm{m}$. The field scattered from a single subwavelength cylinder $n$ can then be written as, ${ }^{18,20}$

$$
E_{n}^{\text {scatt }}(\mathbf{r})=\alpha_{z z} E_{\text {in }}\left(\mathbf{r}_{n}\right) k^{2} G_{0}\left(\mathbf{r}, \mathbf{r}_{n}\right),
$$

where $G_{0}\left(\mathbf{r}, \mathbf{r}_{n}\right)=(i / 4) H_{0}\left(k\left|\mathbf{r}-\mathbf{r}_{n}\right|\right)$ is the free-space Green's function $\left(H_{0}\right.$ is the Hankel function) and $E_{\text {in }}\left(\mathbf{r}_{n}\right)$ is the total incoming field on a given cylinder obtained self-consistently from the solution of

$$
E_{\mathrm{in}}\left(\mathbf{r}_{n}\right)=E_{\mathrm{inc}}\left(\mathbf{r}_{n}\right)+k^{2} \alpha_{z z} \sum_{m \neq n} E_{\mathrm{in}}\left(\mathbf{r}_{m}\right) G_{0}\left(\mathbf{r}_{n}, \mathbf{r}_{m}\right)
$$

In the small particle limit, the polarizability $\alpha_{\mathrm{zz}}$ is given by 19,20 by ${ }^{19,20}$
Downloaded 28 Apr 2009 to 161.111.235.57. Redistribution subject to AlP license or copyright; see http://apl.aip.org/apl/copyright.jsp

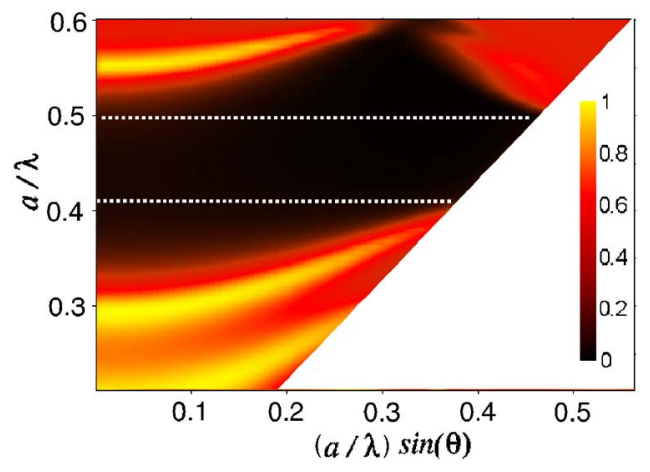

FIG. 2. (Color online) Ratio of transmitted to the incident power for a finite slab of a square lattice $(4 \times 60)$ of Si rods in a map of frequency $(a / \lambda)$ vs transversal momentum of the incoming radiation $(a / \lambda) \sin \theta_{0}$.

$$
\alpha_{z z} \approx \pi R^{2}(\epsilon-1)\left[1-i \frac{\pi}{4}(k R)^{2}(\epsilon-1)\right]^{-1} .
$$

The frequency dependence of the dielectric constant $\epsilon(\omega)$ for $\mathrm{Si}$ is taken into account. ${ }^{21}$

Figure 2 displays the ratio of transmitted to the incident power for a given incident angle for a finite slab of a square lattice of $(4 \times 60) \mathrm{Si}$ rods without PCW (i.e., $W=0)$ in a map of transversal momentum of the incoming radiation versus frequency. Even for a finite slab with only four rows, the transmission map shows a full band gap $(0.4 \lesssim a / \lambda \lesssim 0.5)$ in full agreement with the gap obtained from band structure calculations.

The conductance of the PCW is related to the scattering properties of the system through, ${ }^{22} G=\operatorname{Trace}\left\{\mathbf{t t}^{\dagger}\right\}$, where $\mathbf{t}$ is the transmission matrix of the system. $G$ is usually written as $G=\sum_{i} T_{i}, T_{i}$ being the total transmission for a given incident channel $i$ and the sum runs over all possible incoming channels. For ideal waveguide contacts, adiabatically coupled to reservoirs, $T_{i}$ is either one or zero and the conductance is simply a measure of the number of propagating modes inside the waveguide. In general, $G$ depends on the mechanisms by which light is coupled into and guided through the waveguide. When the incident waves are not confined, i.e., in the continuous limit, the conductance of a two-dimensional waveguide can be written as,

$$
G=\operatorname{Trace}\left\{\mathbf{t t}^{\dagger}\right\}=\frac{k}{2 \pi} \int_{-1}^{+1} \sigma\left(k, \theta_{0}\right) d\left(\sin \theta_{0}\right),
$$

where $\sigma\left(k, \theta_{0}\right)$ is the ratio of transmitted power to incident flux, i.e., the transmission cross section. $\sigma\left(k, \theta_{0}\right)$ can be easily calculated for our finite system provided $W \ll W_{0} \ll M a$, i.e., when the incoming flux of the focused Gaussian beam is uniform over the entrance of the PCW and the illuminated length is smaller than the lateral size of the finite PC. In practice, the calculated cross section is independent of the width of the beam for $W_{0} / \lambda \gtrsim 3 a / \lambda$. In our system, the quantized nature of the optical conductance manifests itself in the behavior of $G$ as a function of the PCW width at fixed frequency. This is illustrated in Fig. 3, where the conductance presents sharp steps at the onset of every propagating mode, reflecting the universal nature of CQ. The conductance behavior is strikingly similar to the one obtained for abrupt wide-narrow-wide electronic constrictions. ${ }^{23}$ While the lowest propagating mode opens at $W=W_{\text {min }} \approx 0.22 \lambda$, higher 


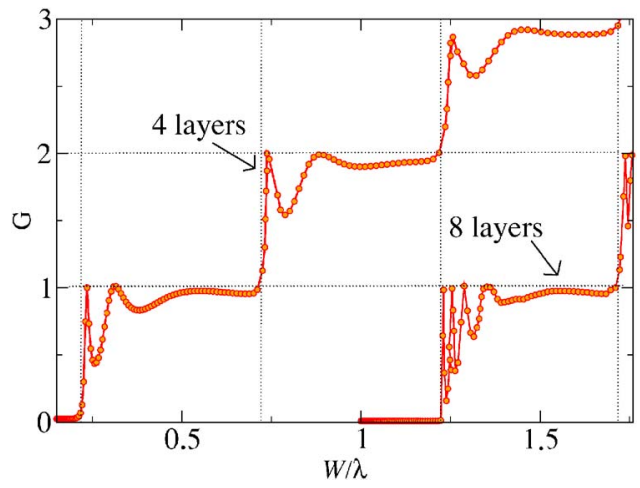

FIG. 3. (Color online) Conductance $G$ vs PCW width $W / \lambda$ at fixed $\lambda$ $=1.33 \mu \mathrm{m}$ for two different PCs (with $N=60$ and $M=4$ and 8). The results for $M=8$ are shifted horizontally for clarity.

This is exactly the expected behavior for a waveguide with perfectly reflected walls.

To summarize, we have introduced the concept of optical conductance in order to characterize the transport properties of waveguides built into photonic crystals. The optical conductance of a waveguide can be obtained experimentally by integrating the transmission cross section as a function of the incoming angle. The measurement of the conductance would provide a direct measure of the number of guided modes supported by the system.

The authors thank E. Sahagún, M. Laroche, R. GómezMedina, and P. García-Mochales for interesting discussions. This work has been supported by the Spanish MCyT (Ref. No. FIS2005-05137 and FIS2006-11170-C02-02), Microseres-CM, and the EU-IP "Molecular Imaging" (LSHG-CT-2003-503259).

${ }^{1}$ E. Yablonovitch, Phys. Rev. Lett. 58, 2059 (1987); S. John, ibid. 58, 2486 (1987); K. M. Ho, C. T. Chan, and C. M. Soukoulis, ibid. 65, 3152 (1990).

${ }^{2}$ Photonic Crystals and Light Localization in the 21st Century, NATO Advanced Studies Institute, Series C: Mathematical and Physical Sciences, edited by C. M. Soukoulis (Kluwer, Dordrecht, 2001), Vol. 563.

${ }^{3}$ J. D. Joannopoulos, R. D. Meade, and J. N. Winn, Photonic Crystals: Molding the Flow of Light (Princeton University Press, Princeton, NJ, 1995).

${ }^{4}$ S. Fan, J. Winn, A. Devenyi, J. C. Chen, R. D. Meade, and J. D. Joannopoulos, J. Opt. Soc. Am. B 12, 1267 (1995); S. G. Johnson, P. R.
Villeneuve, S. Fan, and J. D. Joannopoulos, Phys. Rev. B 62, 8212 (2000); M. Notomik, A. Yamada, A. Shinya, J. Takahashi, C. Takahashi, and I. Yokohama, Phys. Rev. Lett. 87, 253902 (2001).

${ }^{5}$ R. D. Meade, A. Devenyi, J. D. Joannopoulos, O. L. Alerhand, D. A. Smith, and K. Kash, J. Appl. Phys. 75, 4753 (1994); A. Mekis, J. C. Chen, I. Kurland, S. Fan, P. R. Villeneuve, and J. D. Joannopoulos, Phys. Rev. Lett. 77, 3787 (1996); A. Mekis, S. Fan, and J. D. Joannopoulos, Phys. Rev. B 58, 4809 (1998); S. Y. Lin, E. Chow, V. Hietala, P. R. Villeneuve, and J. D. Joannopoulos, Science 282, 274 (1998); T. Baba, N. Fukaya, and J. Yonekura, Electron. Lett. 35, 654 (1999); A. Chutinan and S. Noda, Phys. Rev. B 62, 4488 (2000).

${ }^{6}$ B. J. van Wees, H. van Houten, C. W. J. Beenakker, J. G. Williamson, L. Kouwenhoven, D. van der Marel, and C. T. Foxon, Phys. Rev. Lett. 60, 848 (1988); D. A. Wharam, T. J. Thornton, R. Newbury, M. Pepper, H. Ahmed, J. E. F. Frost, D. G. Hasko, D. C. Peacock, D. A. Ritchie, and G. A. C. Jones, J. Phys. C 21, L209 (1988).

${ }^{7}$ Nanowires, NATO Advanced Studies Institute, Series E: Applied Science, edited by P. A. Serena and N. García (Kluwer, Dordrecht, 1997), Vol. 340; N. Agraï, A. Levy-Yeyati, and J. M. van Ruitenbeek, Phys. Rep. 377, 81 (2003).

${ }^{8}$ E. A. Montie, E. C. Cosman, G. W. T. Hooft, M. B. Van der Mark, and C. W. J. Beenakker, Nature (London) 350, 594 (1991).

${ }^{9}$ C. J. M. Smith, H. Benisty, S. Olivier, M. Rattier, C. Weisbuch, T. F. Krauss, R. M. De La Rue, R. Houdr, and U. Oesterle, Appl. Phys. Lett. 77, 2813 (2000); S. Olivier, M. Rattier, H. Benisty, C. Weisbuch, C. J. M. Smith, R. M. De La Rue, T. F. Krauss, U. Oesterle, and R. Houdré, Phys. Rev. B 63, 113311 (2001).

${ }^{10}$ T. Søndergaard and K. H. Dridi, Phys. Rev. B 61, 15688 (2000).

${ }^{11}$ M. Sigalas, C. M. Soukoulis, E. N. Economou, C. T. Chan, and K. M. Ho, Phys. Rev. B 48, 14121 (1993).

${ }^{12}$ S. G. Johnson, S. Fan, P. R. Villeneuve, J. D. Joannopoulos, and L. A. Kolodziejski, Phys. Rev. B 60, 5751 (1999).

${ }^{13}$ L. Mendioroz, R. Gonzalo, and C. del Rio, Microwave Opt. Technol. Lett. 30, 81 (2001).

${ }^{14}$ M. J. A. de Dood, E. Snoeks, A. Moroz, and A. Polman, Opt. Quantum Electron. 34, 145 (2002).

${ }^{15}$ V. Poborchii, T. Tada, and T. Kanayama, J. Appl. Phys. 91, 3299 (2002).

${ }^{16}$ D. N. Chigrin, A. V. Lavrinenko, and C. M. Sotomayor-Torres, Opt. Express 12, 617 (2004).

${ }^{17}$ H. Giovannini, M. Saillar, and A. Sentenac, J. Opt. Soc. Am. A 15, 1182 (1998).

${ }^{18}$ D. Felbacq, G. Tayeb, and D. Maystre, J. Opt. Soc. Am. A 11, 2526 (1994); E. Centeno and D. Felbacq, Opt. Commun. 160, 57 (1999).

${ }^{19}$ B. T. Draine, Astrophys. J. 333, 848 (1988).

${ }^{20}$ M. Laroche, S. Albaladejo, R. Gómez-Medina, and J. J. Sáenz, Phys. Rev. B 74, 245422 (2006).

${ }^{21}$ D. F. Edwards, Handbook of Optical Constants of Solids, edited by E. D. Palik (Academic, New York, 1985), Vol. 1, p. 547.

${ }^{22}$ E. N. Economou and C. M. Soukoulis, Phys. Rev. Lett. 46, 618 (1981); 47, 973 (1981).

${ }^{23}$ A. Szafer and A. D. Stone, Phys. Rev. Lett. 62, 300 (1989); J. A. Torres and J. J. Sáenz, J. Phys. Soc. Jpn. 73, 2182 (2004). 